\title{
Infrared behavior and infinite-volume limit of gluon and ghost propagators in Yang-Mills theories
}

\author{
Attilio Cucchieri \\ E-mail: attiliodifsc.usp.or \\ Tereza Mendes* \\ E-mail: mendes@ifsc.usp.br \\ Instituto de Física de São Carlos, Universidade de São Paulo, \\ Caixa Postal 369, 13560-970 São Carlos, SP, Brazil
}

\begin{abstract}
Lattice studies of the infrared behavior of gluon and ghost propagators are a key probe of confinement scenarios in Yang-Mills theories. However, finite-volume effects become an important issue as the infrared limit is approached. By considering general quantities — namely an associated susceptibility in the gluon case and properties of the lowest-lying eigenmode of the Faddeev-Popov matrix in the ghost case - one can derive rigorous upper and lower bounds for the propagators. The bounds allow a better control over the extrapolation of lattice results to the infinite-volume limit. In the case of the gluon propagator, an intuitive statistical argument suggests a precise volume dependence for the bounds. This dependence is nicely confirmed by the lattice data, leading to a finite gluon propagator at zero momentum. At the same time, an enhancement of the ghost propagator in the infrared limit seems unlikely. Our analysis is applied to the case of Landau gauge and SU(2) gauge group, using the largest lattice sizes to date.
\end{abstract}

8th Conference Quark Confinement and the Hadron Spectrum September 1-6 2008

Mainz, Germany

${ }^{*}$ Speaker. 


\section{Introduction}

Confinement in QCD and in Yang-Mills theories in general is associated with long-range (infrared) effects. It is thus necessary to study the infrared behavior of the theory's Green's functions using nonperturbative methods. These studies include numerical (lattice) as well as analytic methods and consider basic (gauge-dependent) quantities — such as gluon and ghost propagators in order to test the predictions of the so-called confinement scenarios (see e.g. [1] and references therein). In the case of lattice simulations, one has at one's disposal a true first-principles method, with no uncontrolled approximations. On the other hand, extreme care must be taken to extract the true infrared behavior of the propagators from lattice data, since significant systematic errors may affect the extrapolations that are needed in order to get physical results. The most important such errors are Gribov-copy effects ${ }^{1}$ - related to the fact that the relevant objects are gauge-dependent quantities - and finite-size effects. The latter is especially important in the investigation of the infrared limit, since the smallest nonzero momentum that can be represented on a lattice of linear extension $L$ is proportional to $1 / L$. Thus, a sensible range of small momenta can only be properly simulated on a very large lattice. Here, we consider carefully the elimination of finite-size effects through a better control of the extrapolation of our data to the infinite-volume limit, as described below.

The extrapolation of gluon- and ghost-propagator data to infinite lattice volume is a delicate task, since the correct volume dependence of the data may not be easily inferred from the behavior on medium-size lattices and since some quantities, such as the zero-momentum gluon propagator, are quite noisy. For these reasons it proves very helpful to obtain constraints on the infrared behavior of the propagators, as the upper and lower bounds presented here. We remark that these bounds are valid at each lattice volume $V$ and must be extrapolated to infinite volume, just as for the propagators. The additional advantage, besides establishing a range of allowed values for the propagators, is that the bounds are written in terms of "friendly" quantities - i.e. easier to compute, better behaved or more intuitive than the propagators themselves. It will therefore be more convenient to study the volume dependence of the bounds first, in order to assess the volume dependence of the propagators. We describe and apply the gluon and ghost bounds - in pure $S U(2)$ theory and Landau gauge - respectively in Sections 2 and 3 below. As can be seen from our analysis, we obtain a finite nonzero gluon propagator and a tree-level-like ghost propagator in the infrared limit. Possible implications of these results for the currently accepted confinement scenarios are discussed in the Conclusions.

\section{Gluon Bounds}

Rigorous upper and lower bounds have been introduced in Ref. [6] for the gluon propagator at zero momentum, defined as

$$
D(0)=\frac{V}{d\left(N_{c}^{2}-1\right)} \sum_{\mu, b}\left\langle\left|\widetilde{A}_{\mu}^{b}(0)\right|^{2}\right\rangle
$$

\footnotetext{
${ }^{1}$ The problem of Gribov-copy effects has been extensively studied on the lattice $[2,3,4,5]$. We comment briefly on this issue in our Conclusions.
} 
where $\widetilde{A}_{\mu}^{b}(p)$ is the Fourier transform of the gluon field $A_{\mu}^{b}(x)$ in pure $S U\left(N_{c}\right)$ gauge theory, $\langle\cdot\rangle$ stands for the path integral (Monte Carlo) average, $V=N^{d}$ is the lattice volume and we consider $d$ space-time dimensions. Let us define the quantity

$$
M(0)=\frac{1}{d\left(N_{c}^{2}-1\right)} \sum_{b, \mu}\left|\widetilde{A}_{\mu}^{b}(0)\right| .
$$

It is straightforward to show that this quantity is related to $D(0)$ as

$$
V\langle M(0)\rangle^{2} \leq D(0) \leq V d\left(N_{c}^{2}-1\right)\left\langle M(0)^{2}\right\rangle,
$$

which provides us with rigorous upper and lower bounds for $D(0)$ that must be satisfied at every volume $V$. We can now try to interpret the quantities $\langle M(0)\rangle^{2}$ and $\left\langle M(0)^{2}\right\rangle$, to obtain perhaps an understanding of their volume dependence. We start by noting that if we take the above "magnetization" without the absolute value, i.e. considering

$$
M^{\prime}(0)=\frac{1}{d\left(N_{c}^{2}-1\right)} \sum_{b, \mu} \widetilde{A}_{\mu}^{b}(0)
$$

we get a null Monte Carlo average: $\left\langle M^{\prime}(0)\right\rangle=0$. Because of the absolute value, the quantity defined in Eq. (2.2) has a nonzero average at finite $V$, but it should go to zero at least as fast as $V^{-1 / d}$, as shown in [7]. We now note that $V\left\langle M(0)^{2}\right\rangle$ is essentially the susceptibility associated with the magnetization $M^{\prime}(0)$ (since the average of this magnetization is zero). For a $d$-dimensional spin system one thus expects to see $V\left\langle M(0)^{2}\right\rangle \sim$ const, i.e. the statistical variance of the magnetization is proportional to the inverse of the volume, a behavior known as self-averaging. At the same time, considering the statistical fluctuations in the Monte Carlo sampling of $M(0)$, we would expect $\langle M(0)\rangle^{2}$ to have the same volume dependence as $\left\langle M(0)^{2}\right\rangle[6]$.

The simple statistical argument presented above suggests that both $\langle M(0)\rangle^{2}$ and $\left\langle M(0)^{2}\right\rangle$ should show a volume dependence as $1 / V$, implying (for $d>2$ ) a much stronger approach to zero than the limiting behavior for $M(0)$ obtained in [7] and mentioned earlier. On the other hand, the suppression with $1 / V$ is compensated by the volume factor for both bounds in Eq. (2.3). Consequently, if this suggested behavior for the susceptibilities is verified, $D(0)$ converges to a nonzero constant in the infinite-volume limit. Note that the bounds in Eq. (2.3) apply to any gauge and that they can be immediately extended to the case $D(p)$ with $p \neq 0$.

We have investigated the volume dependence of the bounds for pure $S U(2)$ gauge theory in Landau gauge, considering physical lattice volumes of up to $a^{4} V \approx(27 \mathrm{fm})^{4}$. We find remarkably good agreement with the predicted $1 / V$ behavior for $\langle M(0)\rangle^{2}$ and $\left\langle M(0)^{2}\right\rangle$, as can be seen in plots and tables in Ref. [6]. More precisely, by fitting the two quantities to $1 / V^{\alpha}$ we get the exponents $\alpha$ respectively $0.995(10)$ and $0.998(10)$. Analogously, an analysis for the $S U(3)$ case (considering somewhat smaller volumes) yields the exponents 1.058(6) and 1.056(6) [8]. A similar behavior is also obtained by a study introducing a modified gauge-fixing procedure (in order to check for possible Gribov-copy effects) [9]. Finally, a finite nonzero gluon propagator has been recently obtained using improved actions and anisotropic lattices [10]. We remark that this behavior has also been clearly observed on very large lattices in $3 d[11,6]$ but not in $2 d[12,6]$. 


\section{Ghost Bounds}

Rigorous lower and upper bounds for the ghost propagator $G(p)$ were proposed in [13]. We recall that $G(p)$ is given by the inverse of the Faddeev-Popov (FP) matrix $\mathscr{M}$ and that an infrared enhancement of $G(p)$ with respect to the tree-level ghost propagator $G(p) \sim p^{-2}$ is generally expected as a sign of confinement. By straightforward calculations - independent of the ones performed in the gluon case - we can establish bounds for the ghost propagator. In Landau gauge, for any nonzero momentum $p$, one finds

$$
\frac{1}{N_{c}^{2}-1} \frac{1}{\lambda_{\min }} \sum_{a}\left|\widetilde{\psi}_{\min }(a, p)\right|^{2} \leq G(p) \leq \frac{1}{\lambda_{\min }}
$$

where $\lambda_{\min }$ is the smallest nonzero eigenvalue of the FP operator $\mathscr{M}$ and $\widetilde{\psi}_{\min }(a, p)$ is the corresponding eigenvector. Note that the upper bound is independent of the momentum $p$. If we now assume $\lambda_{\min } \sim L^{-v}$ and $G(p) \sim p^{-2-2 \kappa}$ at small $p$, we have that $2+2 \kappa \leq v$, i.e. $v>2$, is a necessary condition for the infrared enhancement of $G(p)$. A similar analysis can be carried out [14] for a generic gauge condition. Consider the Gribov region $\Omega$, where all eigenvalues of $\mathscr{M}$ are positive. In the infinite-volume limit, entropy favors configurations near the Gribov horizon $\partial \Omega$ (where $\lambda_{\min }$ goes to zero). Thus, inequalities such as (3.1) can tell us if one should expect an enhancement $G(p)$ when the Boltzmann weight gets concentrated ${ }^{2}$ on $\partial \Omega$.

Our study in the $S U$ (2) Landau case [13], using the very large lattices mentioned in the previous section, suggests that $v<2$ (for $d=4$ ). This tree-level-like behavior is confirmed if one considers the dressing function $p^{2} G(p)$. Indeed, the data for the dressing function can be well fitted by $a-b \log \left(1+c p^{2}\right)$ [13], supporting $\kappa=0$. This is also observed in $d=3$. For $d=2$ enhancement is observed, with a behavior $\sim p^{-2 \kappa}$ and $\kappa$ between 0.1 and $0.2[12,13]$.

\section{Conclusions}

By using rigorous upper and lower bounds to constrain the infrared behavior of gluon and ghost propagators, we obtain a finite nonzero gluon propagator at zero momentum and an essentially constant ghost dressing function in the infrared limit. These results seem to contradict the commonly accepted confinement scenarios of Gribov-Zwanziger and Kugo-Ojima [16]. However, as pointed out in [17], the above results are not completely in disagreement with the Gribov-Zwanziger approach. In particular, it has been recently shown [18] that using the Gribov-Zwanziger approach, i.e. by restricting the functional integration to the Gribov region $\Omega$, one can also obtain in $3 d$ and $4 d$ a finite nonzero gluon propagator and a tree-level-like ghost propagator in the infrared limit. It is interesting that the same approach cannot be applied to the $2 d$ case [19]. Let us also note [17] that even though the Gribov-Zwanziger and the Kugo-Ojima confinement scenarios seem to predict similar infrared behavior for the propagators, it is not clear how to relate the (Euclidean) cutoff at the Gribov horizon to the (Minkowskian) approach of Kugo-Ojima [20].

Similar results for the gluon and ghost propagators are obtained by various groups using very large lattice volumes [21], both in the $S U(2)$ and in the $S U(3)$ cases. [The equivalence between the

\footnotetext{
${ }^{2}$ For example, in $4 d$ Maximally Abelian gauge one sees that $\lambda_{\min }$ goes to zero at large volume but the ghost propagator stays finite at zero momentum [15].
} 
infrared propagators in $S U(2)$ and $S U(3)$ gauge theories can be seen e.g. in [22].] Of course, one should also recall that the region $\Omega$ is actually not free of Gribov copies and that the configuration space should be identified with the so-called fundamental modular region $\Lambda$. On the other hand, the restriction to $\Lambda$ and the numerical verification of the Gribov-Zwanziger scenario are separate issues [17]. Indeed, this scenario is based on the restriction of the configuration space to the region $\Omega$, which includes $\Lambda$. Finally, as explained in [2], the restriction to $\Lambda$ can only make the ghost propagator less singular, as confirmed by recent lattice data [5].

Acknowledgements The authors acknowledge partial support from the Brazilian Funding Agencies FAPESP and CNPq. T.M. also thanks the Theory Group at DESY-Zeuthen for hospitality and the Alexander von Humboldt Foundation for financial support.

\section{References}

[1] A. Maas, A. Cucchieri and T. Mendes, Braz. J. Phys. 37N1B, 219 (2007).

[2] A. Cucchieri, Nucl. Phys. B 508, 353 (1997).

[3] A. Cucchieri, Nucl. Phys. B 521, 365 (1998).

[4] P. J. Silva and O. Oliveira, Nucl. Phys. B 690, 177 (2004).

[5] A. Maas, arXiv:0808.3047 [hep-lat].

[6] A. Cucchieri and T. Mendes, Phys. Rev. Lett. 100, 241601 (2008).

[7] D. Zwanziger, Phys. Lett. B 257, 168 (1991).

[8] O. Oliveira and P.J. Silva, arXiv:0809.0258 [hep-lat].

[9] I. L. Bogolubsky et al., Phys. Rev. D 77, 014504 (2008) [Erratum-ibid. D 77, 039902 (2008)]; V. G. Bornyakov, V. K. Mitrjushkin and M. Muller-Preussker, arXiv:0812.2761 [hep-lat].

[10] M. Gong, Y. Chen, G. Meng and C. Liu, arXiv:0811.4635 [hep-lat].

[11] A. Cucchieri, T. Mendes and A. R. Taurines, Phys. Rev. D 67, 091502 (2003).

[12] A. Maas, Phys. Rev. D 75, 116004 (2007).

[13] A. Cucchieri and T. Mendes, arXiv:0804.2371 [hep-lat].

[14] A. Cucchieri, AIP Conf. Proc. 892, 22 (2007).

[15] T. Mendes, A. Cucchieri and A. Mihara, AIP Conf. Proc. 892, 203 (2007); T. Mendes, A. Cucchieri, A. Maas and A. Mihara, arXiv:0809.3741 [hep-lat].

[16] A. Cucchieri and T. Mendes, Braz. J. Phys. 37 (2007) 484.

[17] A. Cucchieri and T. Mendes, arXiv:0809.2777 [hep-lat].

[18] D. Dudal et al., Phys. Rev. D 77, 071501 (2008); arXiv:0806.4348 [hep-th]; arXiv:0808.0893 [hep-th].

[19] D. Dudal, S.P. Sorella, N. Vandersickel and H. Verschelde, arXiv:0808.3379 [hep-th].

[20] D. Zwanziger, Phys. Rev. D 69, 016002 (2004).

[21] A. Cucchieri and T. Mendes, PoS LAT2007, 297 (2007); I.L. Bogolubsky, E.M. Ilgenfritz, M. Muller-Preussker and A. Sternbeck, PoS LAT2007, 290 (2007); A. Sternbeck, L. von Smekal, D.B. Leinweber and A.G. Williams, PoS LAT2007, 340 (2007).

[22] A. Cucchieri, T. Mendes, O. Oliveira and P.J. Silva, Phys. Rev. D 76, 114507 (2007). 